\title{
Cloud Computing in Agricultural Development of Rural India
}

\author{
Subhendu Chatterjee
}

Department of Computer Science, Gour Mahavidyalaya, Mangalbari, Malda, West Bengal, India

Corresponding author: subhmaha1@gmail.com

Received: $16-01-2020$

Revised: $22-04-2020$

Accepted: 27-05-2020

\begin{abstract}
Now India lives in its villages and smaller towns and in that respect lies the future of India. Indian villages have been ignored for more than 72 years. Now the cloud computing technology will bring the changes that were required to bridge the rural India and Urban India. This will enhance the Indian rural economy as well as country's economy. The paramount sources of income of Indian people are agriculture. Therefore, the development of the ICT is basically focused on the Indian agriculture field. Cloud computing technology is a general term which is used to describe a new class of network based computing that take place via the internet. These platforms hide the complication and details of elemental infrastructure from users and applications by providing very simple graphical interface. Latest Cloud computing technology impacted positively on agriculture field and related services they provide for users.
\end{abstract}

Keywords: Cloud computing, Community model, Hybrid model, Public model, Private model, Agriculture, IaaS, Paas \& SaaS

Agriculture is the main source of income sector in India and the ICT application of cloud computing plays comprehensive role to augment the production in agriculture [1]. ICT bear well customized, updated and secured database with instantaneous connectivity at legitimate investment cost. These facilities provides a lots of facilities like real time computation, data access and storage to the farmers without getting aware of physical location and configuration of the system [2]. An agro advisory system is imported in Gujarat region of India that consists of three basic components, which are: cotton ontology, web services and mobile application development [2]. Farmers can access their queries from cotton ontology respecting cotton soil, horticulture process, disease, pest and other relevant information. Web services settled the 
communication with different data sources and all these applications which are available on simple android phones.

\section{Objective}

The main objective of my paper is thus concerned with the perception of cloud Computing and its adequate implementation as well as how adequately it can devote as an ICT tool to play a crucial role in developing the Indian agricultural sectors.

\section{Cloud Computing}

Cloud computing gets its name as a metaphor for the Internet. Typically, the Internet is represented in network diagrams as a cloud. The cloud icon represents "all that other stuff" that makes the network work. Cloud computing is an on-demand service model for Information Technology services, often based on virtualization and distributed computing technologies. Cloud computing architectures have:

Hugely abstracted resources.

Instant scalability and flexibility

Instantaneous provisioning

Shared resources like hardware, database, memory, etc.

"Service on demand", Usually with a billing system

* Programmatic management like through WS API.

We should scramble to the cloud because there is valid and significant business and IT reasons for the cloud computing paradigm shift. The fundamentals of outsourcing as a solution apply:

* Reduced cost: Cloud computing can reduce both capital expense (CapEx) and operating expense $(\mathrm{OpEx})$ costs because resources are only acquired when needed and are only aid for when used.

* Refined usage of personnel: Using cloud computing frees valuable personnel allowing them to focus on delivering value rather than maintaining hardware and software.

Robust scalability: Cloud computing allows for immediate scaling, either up or down, at any time without long-term commitment [3].

\section{Categories of Cloud Computing Infrastructure}

Cloud computing infrastructure is the collection of hardware as well as software elements needed to enable cloud computing. It includes computing power, networking, and storage including an interface for users to access their virtualisation resources. The virtual resources are the mirror of a physical infrastructure, with components like servers, network switches, memory and storage clusters.

(a) Software as a service (SaaS): This software offered by a third party provider, available on demand, usually via the Internet configurable remotely that include online word processing and spreadsheet tools, 
CRM services and web content delivery services. Software as a Service (SaaS) is traditionally comes to mind when we think about cloud computing. In SaaS, an application is hosted by a service provider and then accessed via the WWW by a client. A cloud consumer releases his applications on a hosting environment, which can be accessed through networks from various clients by application users. Cloud consumers do not have control over the Cloud infrastructure that often employs multi-tenancy system architecture, namely, different cloud consumers' applications are organized in a single logical environment on the SaaS cloud to achieve economies of scale and optimized in terms of speed, security, availability, disaster recovery, and maintenance. Examples of SaaS include SalesForce.com, Google Mail, Google Docs, and many other [4].

(b) Platform as a service (PaaS): Platform as a Service (PaaS) is one of the application of delivery model. PaaS supplies all the resources to build applications and services completely from the Internet, without having to download or install any software. It allows customers to develop new applications using API deployed and configurable remotely. The platforms offered include development tools, configuration management, and deployment platforms. Examples are Microsoft Azure, Force and Google App engine. PaaS, we have to look at which applications are most appropriate for maintenance on the cloud. It will obviously difference from organization to organization, but we likely won't move our key mission like critical tasks to the cloud. For instance, a company that develops software for healthcare providers is going to have different needs than a financial advisor, for instance. But even within the same industry, different organizations will get different things out of the cloud.

Hence the difference between SaaS and PaaS is that SaaS only hosts completed cloud applications whereas PaaS offers a development platform that hosts both completed and in-progress cloud applications. This requires PaaS, in addition to supporting application hosting environment, for possess development infrastructure including programming environment, tools, configuration management, and so forth. An example of PaaS is Google AppEngine [4].

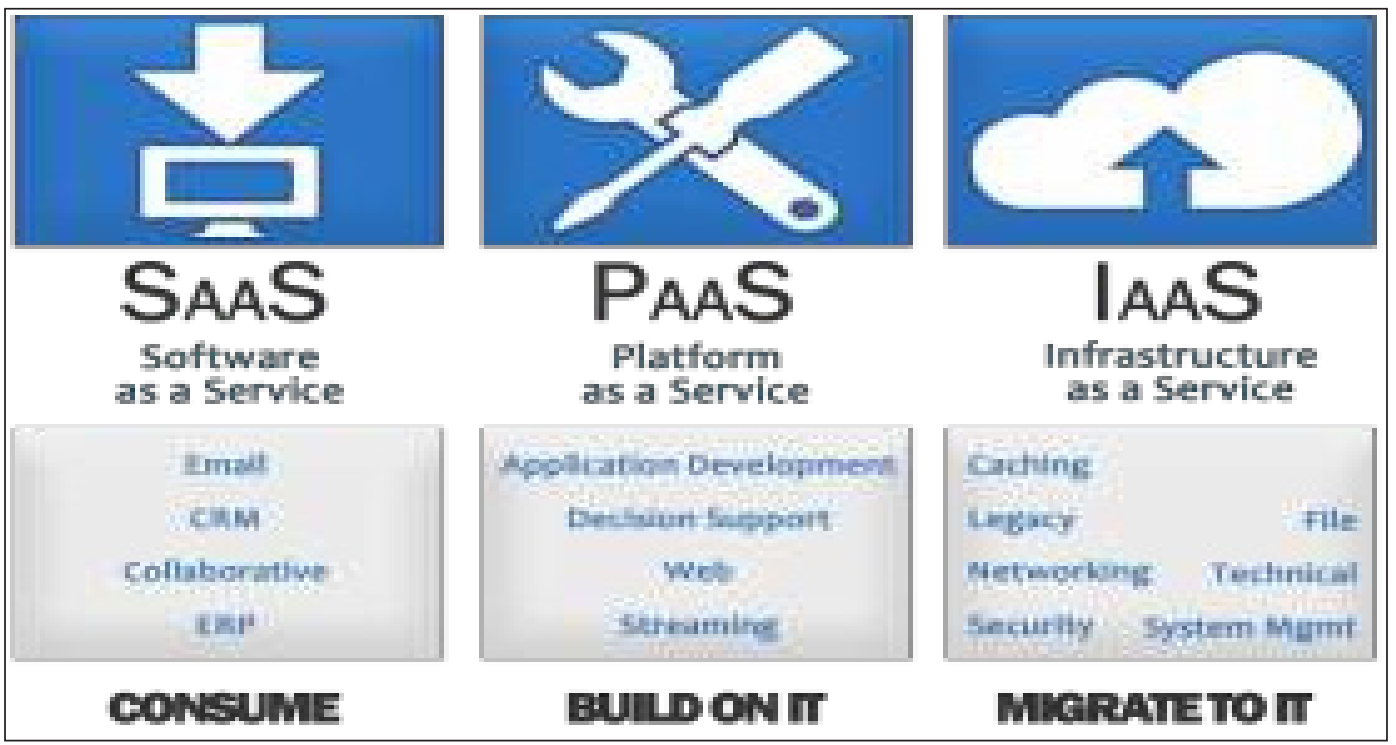

Fig. 1: Basic Architectures of Cloud Infrastructure 
(c) Infrastructure as service (IaaS): It provides virtual machines and other abstracted hardware and OS which may be controlled through a service API. Examples include Amazon EC2 and S3, Terre mark Enterprise Cloud, Windows Live Skydrive and Rackspace Cloud. Hardware as a Service (HaaS) is the next form of service which are available in Cloud computing. Where SaaS and Paas are providing applications customers, Haas doesn't. It simply offers the hardware so that our organization can put whatever they want onto it. The basic strategy of virtualization is to set up independent virtual machines (VM) that are common from both the underlying hardware and other VMs. Notice that this strategy is different from the multi-tenancy model, which aims to transform the application software architecture so that multiple instances can run on a single application. An example of IaaS is Amazon's EC2 [4].

(d) Database as a Service (DaaS): Another service offering that is becoming prevalent in the world of cloud computing is Database as a Service (DaaS). The idea behind DaaS is to avoid the complexity and the running cost of our own database. DaaS allows consumers to pay for what they are actually using rather than the site license for the entire database. In addition to traditional storage interfaces such as RDBMS and other file systems, some DaaS offerings provide table-style abstractions that are designed to scale out to store and retrieve a huge amount of data within a very compressed time-frame, often too large, too expensive as well as too slow for most commercial RDBMS to cope with. Examples of DaaS include Amazon S3, Google BigTable, and Apache HBase, etc. [4] [5].

\section{Characteristics of Cloud Computing [6]}

* On-demand self-service: A customer can unilaterally provision computing capabilities, such as server time and network storage, as needed automatically without fascinating human interaction with each service providers.

* Broad network access: Efficiencies are available over the network \& penetrate through standard mechanisms that affirm use by heterogeneous thick or thin client platforms like mobile phones, tablets, laptops, and workstations etc.

* Rapid elasticity: Cloud services can be rapidly as well as elastically provisioned, in some cases automatically, to quickly and rapidly scale out as well as released to quickly scale in. To the customer, the capabilities are available for provisioning generally appear to be unlimited and can be purchased at any time in any quantity.

Measured service: Pay per use-capabilities is charged using a metered, fee-for-service, or advertising based billing model to boost optimization of resource use. Examples are measuring the storage, bandwidth \& computing resources consumed and charging for the number of active user accounts per month.

* Resource pooling: The provider is computing resources are pooled to serve multiple consumers using a multi-tenant model, with different physical and virtual resources dynamically assigned and reassigned according to consumer demand. There is a sense of location independence in that the customer generally has no control or knowledge over the exact location of the provided resources but may be able to specify location at a higher level of virtuality like country, state, or data center etc. Examples of resources include storage, processing, memory, network bandwidth, and virtual machines. 


\section{Role of Cloud Computing in Rural Agriculture Field [6]}

Agriculture information data bank like crop, weather, soil, growth progress, farmer data \& expert consultation.

* Store all the agriculture related information in a centralized cloud, which will be available to all the users at anywhere, anytime.

Management of all data related to land, location, area; soil and land characteristics through centralized decision support systems

- High integration \& sharing of agricultural information

It can be eliminate the farmer's limitations of technical knowledge \& resources

* Providing agricultural technology service \& science

- Improvement of the agricultural products marketing

* Efficient use of agricultural resources

- Promote the circulation of agricultural product and service in wider level.

\section{Benefits of Cloud Computing in Agriculture [6]}

Data Readiness any time \& any where

* Local and global communication

* Improve economic condition of the Nation

* Increase the GDP of the nation

* Ensure food security level

Motivation of farmers and researchers

Reduction of technical issue

Rural-Urban movement

Data availability at any time and at any location without delay

Improve market price of Food, seeds, other product

\section{Challenge of Cloud Computing in Agriculture [6]}

Maintenance \& Supervision by third party, So data security is less

Indirect administrator accountability

Farmer is unknown for cloud computing technology

Less physical control

Attraction to hackers 
Need on the network connectivity

Requires a constant Internet connection

Platform facility is not easily available for farmers

Farmers training necessary for this technology

Does not work well with low-speed connections

It runs the risk of security

\section{Agriculture and Cloud Computing Technology [7]}

India is one of the largest producers of foods, grains and other products, but still agriculture and its production process are decentralized, crude and obsolete methods being followed by the farmers, together with several constraints of the farmers and modernization is very slow. This results in an obvious gap between the supply and demand chains of the agricultural products. This will have a negative impact on the farmer's economic conditions as well the national income of the country. This bottleneck can be eliminated with the implementation of Cloud in agricultural field. It can break the farmer's limitations in technical knowledge, improve the utilization of existing resources and can also overcome the strong dependence on natural climate in the specific geographical areas. By conveying important information related to agriculture through Cloud and other devices through Internet, the farmers can benefit hugely.

\section{Proposed System and Design [7]}

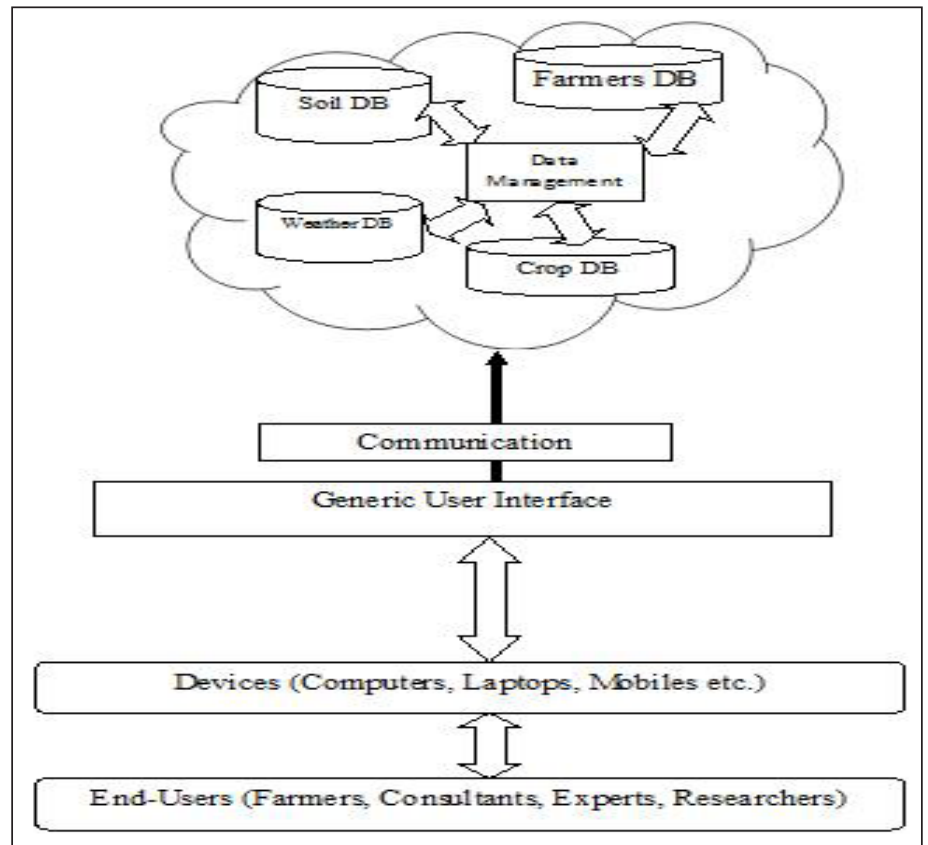

Fig. 2: System design 
The proposed system as shown in Fig. 2, suggests that cloud can be implemented with a model with two core parts:

1. An approach that is user-friendly and fast to monitor and fulfil the user requests- Cloud system

2. A centralized location to store all relevant data-Cloud.

\section{Necessity of Proposed System in Agricultural Sectors [7]}

Cloud Computing technology is recently picking up the global market extensively covering almost all the prime sectors and areas but nothing is still done in the Indian Agro sector. The developed countries have already taken up this project but still in the infant state.

The effective use of Cloud in agriculture sector will bring positive changes by utilizing cloud's key features. The following benefits may serve as reasons as to why cloud technology is necessary to be implemented in the agricultural sector:

* It is an attractive technology from the point of view of cost of setting up. Low startup cost is affordable in rural India.

* Information management (related to crop, soil, and weather and production process) is easier as it will be managed by the service providers, a team of professionals.

* Promotes the circulation of agricultural products. Long supply chain and complex link between farmers and consumers makes it difficult for the farmers to derive benefits and value from the markets.

* Unemployment problem is resolved as services are provided to smallest of the place and however the place is remote.

Data availability at any time and at any location.

Urban rural migration can be reduced.

Technical issues will be reduced as they are handled by service providers and professionals.

\section{CONCLUSION}

Besides numerous applications in various scientific as well as business areas, cloud computing has found its vast applicability in agriculture as well. India will definitely benefit if the proposed model is implemented effectively in the agricultural sector. An effective implementation of this model will encourage other sectors also, which will lead to optimal benefit of shifting towards cloud [8]. This will bridge the gap between technology, information and farmers of India. This will have positive and tremendous impact on other fields also that will lead the nation towards technological development. Therefore, promotion and awareness of Cloud computing technology and its use, implementation will bring out new zenith in economic development in India. Cloud computing technology, applicable for the improvement of agriculture growth, food, grain, product, economic condition, Ensure food safety, GDP of the nation \& circulate information related to agriculture etc. 


\section{REFERENCES}

1. Chen, N., Zhang, X. and Wang, C. 2015. "Integrated open geospatial web service enabled cyberphysical information infrastructure for precision agriculture monitoring," Computer and Electronics in Agriculture, 111: 78-91.

2. Chaudhary, S., Bhise, M., Banerjee, A., Goyal, A. and Moradiya, C 2015. "Agro Advisory System for Cotton Crop," In: Proc $7^{\text {th }}$ International Conference on Communication Systems and Networks, pp. 1-6, January 6-10.

3. Cloud computing fundamentals - A different way to deliver computer resources Skill Level: Introductory Grace Walker IT Consultant Walker Automated Services.

4. Cloud Computing: Issues and Challenges Tharam Dillon Digital Ecosystems and Business Intelligence Institute Curtin University of Technology Perth, Australia tharam.dillon@curtin.edu.au Chen Wu and Elizabeth Chang Digital Ecosystems and Business Intelligence Institute Curtin University of Technology Perth, Australia\{chen.wu, change\}@curtin.edu.au.

5. Huhns, M.N. and Singh, M.P. 2005. "Service-Oriented Computing: Key Concepts and Principles," IEEE Internet Computing, Internet Computing, 09: 75-81.

6. Choudhary, S.K., Jadoun, R.S. and Mandoriya, H.L. 2016. Computer Engineering and Intelligent Systems, ISSN 2222-1719 (Paper) ISSN 2222-2863 (Online), 7(3).

7. Seena Kalghatgi, Kuldeep, P. Sambrekar, 2015. IJISET - International Journal of Innovative Science, Engineering \& Technology, 2(3).

8. Patel, R. and Patel, M. 2013. Lecturer, Department of Information Technology, Kirodimal Institute of Technology Raigarh, "Application of Cloud Computing in Agricultural Development of Rural India”, International Journal of Computer Science and Information Technologies, 4(6). 